\title{
The Influence of a Crosshair Visual Aid on Observer Detection of Simulated Fetal Heart Rate Signals
}

\author{
Rebecca A. Kennedy, $\mathrm{MS}^{1}$ Mark W. Scerbo, $\mathrm{PhD}^{1}$ \\ Lee A. Belfore II, $\mathrm{PhD}^{3}$ Alfred Z. Abuhamad, MD \\ ${ }^{1}$ Department of Psychology, Old Dominion University, Norfolk, \\ Virginia \\ ${ }^{2}$ Carolinas Healthcare System, Matthews, North Carolina \\ ${ }^{3}$ Department of Electrical and Computer Engineering, Old Dominion \\ University, Norfolk, Virginia \\ ${ }^{4}$ Eastern Virginia Medical School, Hofheimer Hall, Norfolk, Virginia
}

Brittany L. Anderson-Montoya, $\mathrm{PhD}^{2}$

Stephen S. Davis, MD 4

\begin{abstract}
Address for correspondence Rebecca A. Kennedy, MS, Department of Psychology, Old Dominion University, Norfolk, VA 23529

(e-mail: becca.kennedy92@gmail.com).
\end{abstract}

Am J Perinatol Rep 2016;6:e115-e120.

\begin{abstract}
Keywords

- maternal-fetal heart rate monitoring

- signal detection

- visual aid

- fetal heart rate

Objective To determine whether a visual aid overlaid on fetal heart rate (FHR) tracings increases detection of critical signals relative to images with no visual aid.

Study Design In an experimental study, 21 undergraduate students viewed 240 images of simulated FHR tracings twice, once with the visual aids and once without aids. Performance was examined for images containing three different types of FHR signals (early deceleration, late deceleration, and acceleration) and four different FHR signal-to-noise ratios corresponding to FHR variability types (absent, minimal, moderate, and marked) identified by the National Institute of Child Health and Human Development (2008). Performance was analyzed using repeated-measures analyses of variance.

Results The presence of the visual aid significantly improved correct detections of signals overall and decreased false alarms for the marked variability condition.

Conclusion The results of the study provide evidence that the presence of a visual aid was useful in helping novices identify FHR signals in simulated maternal-fetal heart rate images. Further, the visual aid was most useful for conditions in which the signal is most difficult to detect (when FHR variability is highest).
\end{abstract}

Changes in fetal heart rate (FHR) during labor can provide important information about the wellbeing of the fetus. However, despite improvements in standardized FHR definitions and a simplification of interpretations, there remains a lack of consensus on a standardized approach for assessing FHR. ${ }^{1}$ There is still a large amount of subjectivity involved in interpreting patterns in FHR tracings, which can unfortunately lead to misinterpretations and thus inadvertent adverse consequences.

In 2008, the National Institute of Child Health and Human Development (NICHD) recommended a three-tier classification system that defines FHR patterns as normal, indeterminate, or abnormal to assist with interpretation. ${ }^{2}$ FHR patterns that fall in the normal category (Category I) are considered reassuring; these tracings can be followed in a routine manner without specific action. Category III patterns represent abnormal patterns, which are regarded as ominous and often require immediate clinical intervention. Finally, indeterminate patterns (Category II) are those that should be closely monitored to assess whether they indicate a problem.

Although the NICHD classification system provides a useful framework for FHR assessment, there is still potential for received

August 26, 2015 accepted after revision

December 9, 2015
DOI http://dx.doi.org/

10.1055/s-0036-1571331. ISSN 2157-6998.
Copyright $\odot 2016$ by Thieme Medical Publishers, Inc., 333 Seventh Avenue, New York, NY 10001, USA. Tel: +1(212) 584-4662.
License terms

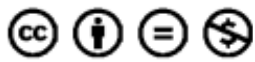


clinicians to misclassify FHR patterns within this structure. Two patterns that may frequently be misinterpreted are early decelerations and late decelerations. Both types of decelerations appear as temporary declines in FHR, but they are characterized by different timing relative to the contraction. Early decelerations generally have their nadir concurrent with the peak of the uterine contraction and are considered reassuring patterns. Late decelerations have their onset after the peak of the contraction and, unlike early decelerations, repeated late decelerations are considered ominous and an indication of fetal hypoxemia. It is important for clinicians to properly distinguish FHR patterns like early decelerations and late decelerations to ensure fetal and maternal wellbeing and to avoid unnecessary clinical intervention.

Using an FHR simulator, ${ }^{3}$ Anderson and colleagues have conducted several studies investigating individuals' abilities to identify FHR signals in static images as well as dynamic tracings. Both untrained undergraduate students and trained professionals were found to have difficulty distinguishing between early and late decelerations. ${ }^{4-6}$ The researchers also found that FHR signals are more difficult to detect when variability increases. $^{7}$

A visual aid has been created to assist individuals with detecting FHR signals. The aid is a large, moveable crosshair consisting of a horizontal bar and a vertical bar that each extend across the tracing. The horizontal bar may help observers detect FHR accelerations and decelerations by providing a visual guide of FHR relative to the baseline. The vertical bar may be helpful by providing a visual guide for determining whether a deceleration is early or late relative to the contraction. The purpose of the current research was to determine whether the presence of the visual aid in static FHR images would improve detection performance.

\section{Materials and Methods}

\section{Participants}

Twenty-one undergraduate students (7 women, 14 men) from Old Dominion University (ODU) with a mean age of 19.33 years $(S D=0.30)$ and no formal training in the interpretation of FHR tracings took part in this study. All participants had normal or corrected-to-normal vision. The ODU Instructional Review Board approved the study.

\section{Fetal Heart Rate Display}

Static images of simulated tracings were produced using the FHR simulator created by Belfore and colleagues. ${ }^{3}$ The images each measured $18 \times 15.5 \mathrm{~cm}$ with a white background and two red grids, one for the FHR and another below for the maternal uterine contractions. Both tracings were displayed in blue. Every image contained a single contraction. Some images contained an FHR acceleration or deceleration to be identified and others contained the FHR tracing without accelerations or decelerations present.

The FHR variability in the tracings was generated mathematically from specific signal-to-noise $(\mathrm{S} / \mathrm{N})$ ratios, where the signal refers to the acceleration or deceleration. Four S/N ratios were generated to approximate the categories designated by the NICHD: absent $(S / N$ ratio $=10: 1)$, minimal $(S / N$ ratio $=5: 1)$, moderate $(S / N$ ratio $=2: 1)$, and marked $(\mathrm{S} / \mathrm{N}$ ratio $=1: 1)$. Depictions of the four $\mathrm{S} / \mathrm{N}$ ratios are displayed in - Fig. $\mathbf{1}$.

The visual aid was a semitransparent turquoise crosshair overlaid on the static image. The horizontal bar of the aid was placed at baseline (defined as the mean of the FHR variability) to assist with the detection of deceleration or acceleration deviations from baseline. The vertical bar was placed at the location of the peak of the contraction to help users distinguish whether FHR decelerations were early or late in nature. - Fig. 2 displays an example of an image that contained the visual aid.

\section{Procedure}

There were three sets of FHR tracing images, each addressing a single type of signal (early deceleration, late deceleration, or acceleration). The signals were represented by small, medium, and large deviations from the FHR baseline (i.e., deviation of 5,10 , or $15 \mathrm{bpm}$ ). For the early deceleration images, the nadir was coincident with the peak of the contraction. For the late deceleration images, the nadir was delayed from the contraction peak by $4,8,12$, or 16 seconds.

Each set contained 48 images in which a signal (i.e., an early deceleration, late deceleration, or acceleration) was present, and 32 images that contained no signals, to ensure that participants were required to determine whether images contained signals at all. Within each set, there were 12 images depicting each NICHD variability type (absent, minimal, moderate, and marked) combined with the three signal sizes (small, medium, and large). The images were presented in two blocks, once with the visual aids and once without the aids for a total of 240 images. The orders of the blocks and sets of images were randomized and counterbalanced across participants.

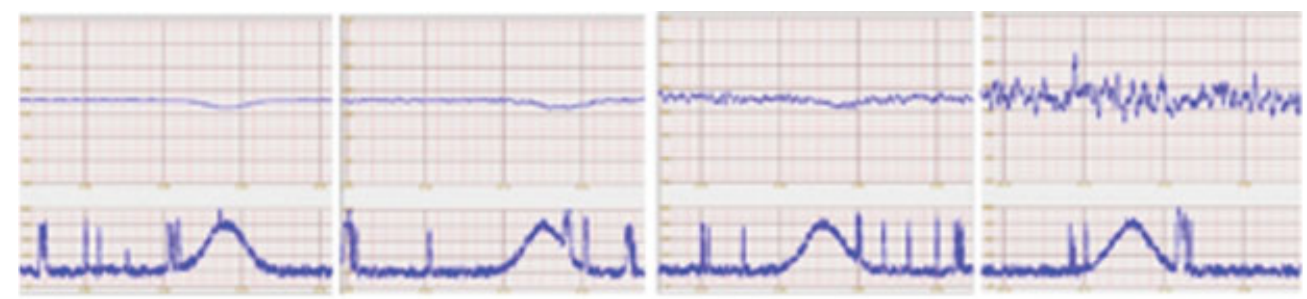

Fig. 1 Examples of simulated fetal heart rate decelerations embedded in four variability categories (absent, minimal, moderate, and marked) increasing from left to right. 


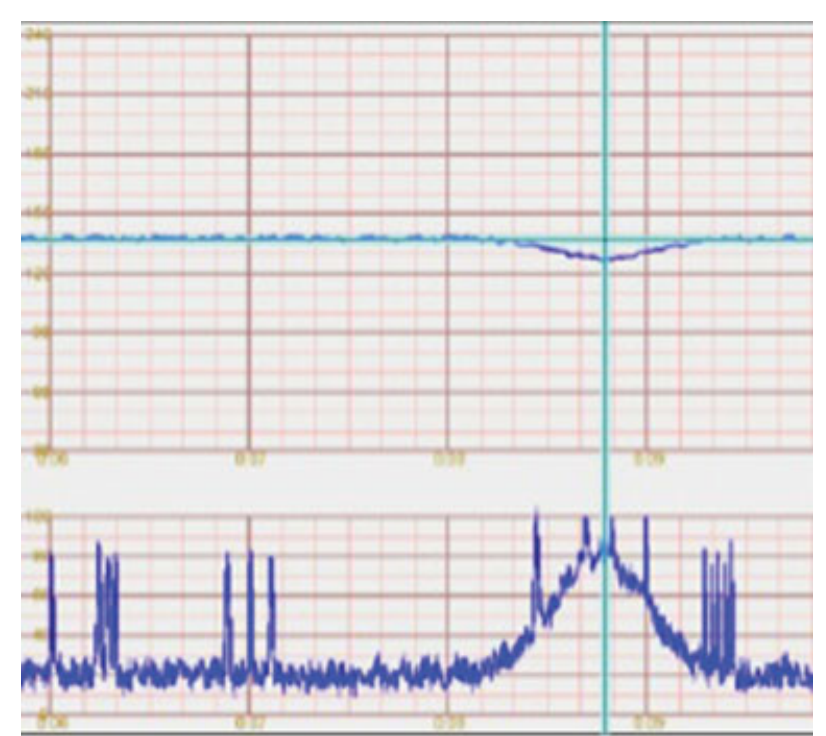

Fig. 2 Example of a visual aid overlay on an early deceleration with minimal variability.

Participants were provided instructions informing them which type of signal they would be looking for in each set. Each image was presented for 3 seconds, after which a response screen required participants to indicate whether a signal was present and, if so, to identify the signal type and magnitude by clicking on a response choice using a mouse. Once participants selected a response, the next image was immediately presented. Participants were given a 10-minute break in between the two blocks.

\section{Data Analysis}

The data were analyzed using a 2 (aid) $\times 3$ (signal type, i.e., early deceleration, late deceleration, acceleration) $\times 4$ (variability; i.e., absent, minimal, moderate, marked) repeatedmeasures analysis of variance. Mauchly test was used to determine violations of the sphericity assumption. If detected, the Greenhouse-Geisser correction was used for the degrees of freedom. The Bonferroni-Sidak method was used for post-hoc tests.

\section{Results}

\section{Proportion of Correct Detections}

Correct detections were defined as the correct identification of an early deceleration, late deceleration, or acceleration in the image. Correct detections were calculated as a proportion of correct responses relative to the actual number of signals that were presented. Correct answers were defined based on NICHD definitions for early and late decelerations and accelerations.

There was significant effect of aid on correct detections, $F$ $(1,20)=8.43, p<0.05$, partial $\eta^{2}=0.292$. The presence of the aid led to a higher rate of detections $(M=0.62, \mathrm{SE}=0.03)$ than the absence of aids $(M=0.53, \mathrm{SE}=0.03)$.

Signal type also significantly impacted correct detections, $F(1.97, \quad 39.44)=4.19, \quad p<0.05, \quad$ partial $\eta^{2}=0.173$.
See - Table 1 for means and standard errors. Correct detections were significantly higher for early decelerations $(M=0.61, \mathrm{SE}=0.03)$ than accelerations $(M=0.54, \mathrm{SE}$ $=0.03$ ). No other differences in correct detections between signal types were significant.

As predicted based on prior research, ${ }^{7}$ there was also a significant effect of variability (i.e., absent, minimal, moderate, marked, based on $\mathrm{S} / \mathrm{N}$ ratios) on correct detections, $F$ $(1.98,39.62)=114.73, p<0.05$, partial $\eta^{2}=0.852$. The means and standard errors are shown in - Table 2. Highest correct detections occurred for absent and minimal variability, with lower correct detections for moderate variability and lowest for marked variability. Therefore, increasing variability resulted in decreased correct detections. All means were significantly different from one another except for the difference between absent and minimal variability, which were equivalent.

There was also a significant interaction for proportion of correct detections between the presence of aids and variability, $F(2.44,48.84)=3.87, p<0.05$, partial $\eta^{2}=0.162$. However, a Bonferroni-Sidak test revealed that none of the differences between signal types according to aid presence were significant (-Fig. $\mathbf{3}$ ).

Finally, there was a significant interaction between signal type (i.e., late deceleration, early deceleration, and acceleration) and variability for proportion of correct detections, $F$ $(4.02,80.49)=4.02, p<0.05$, partial $\eta^{2}=0.167$. A Bonferroni-Sidak test indicated that all differences were significantly different from one another except for the difference between absent and moderate variability for late decelerations. The means and standard errors for this interaction are displayed in - Table 3.

\section{Proportion of False Alarms}

False alarms were defined as instances in which participants incorrectly indicated that a deceleration was present in a tracing that contained none. There was a significant interaction between aid presence and variability for false alarm

Table 1 Mean rate of correct detections according to signal type

\begin{tabular}{|l|l|l|}
\hline Signal type & Mean & Standard error \\
\hline Late deceleration & 0.57 & 0.02 \\
\hline Early deceleration & 0.61 & 0.03 \\
\hline Acceleration & 0.54 & 0.03 \\
\hline
\end{tabular}

Table 2 Mean rate of correct detection according to variability type

\begin{tabular}{|l|l|l|}
\hline Variability type & Mean & Standard error \\
\hline Absent & 0.71 & 0.03 \\
\hline Minimal & 0.73 & 0.02 \\
\hline Moderate & 0.51 & 0.03 \\
\hline Marked & 0.34 & 0.02 \\
\hline
\end{tabular}




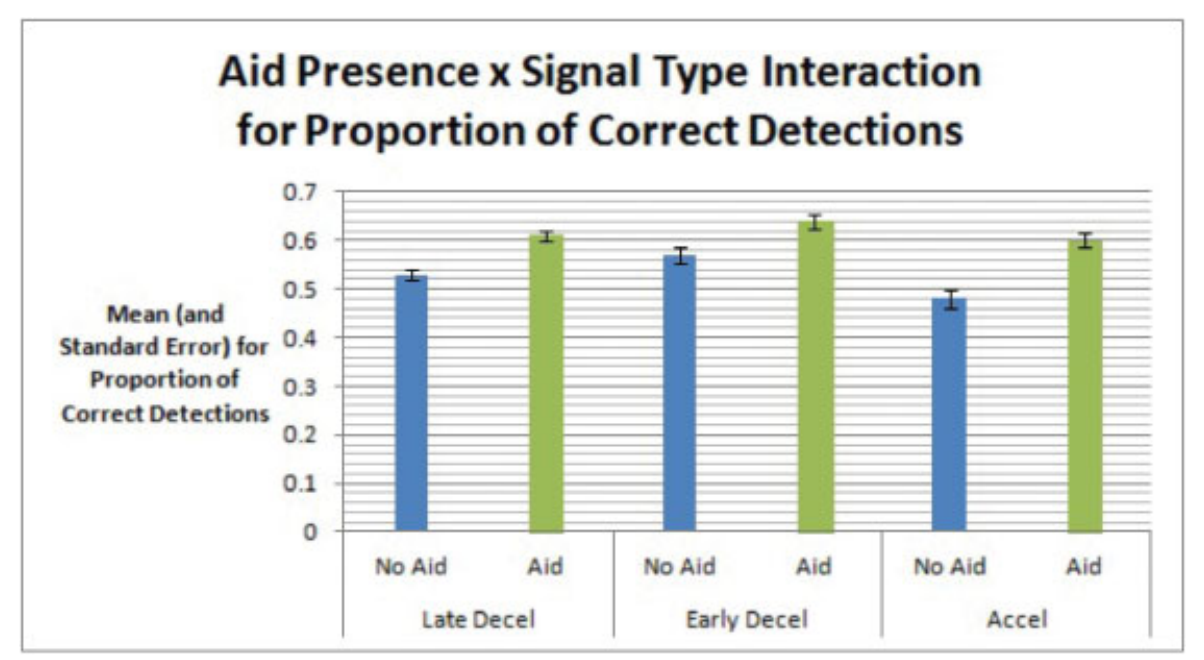

Fig. 3 Aid presence $\times$ signal type interaction for correct detections.

rates, $F(1.97,39.48)=4.92, p<0.05$, partial $\eta^{2}=0.197$. See - Fig. 4 for a graph of means and standard errors of false alarm rate according to aid presence and variability. A Bonferroni-Sidak test showed that there were significant differences between aid presence and aid absence for marked variability only.

\section{Discussion}

The purpose of the study was to examine whether the presence of a visual aid could assist individuals with correctly identifying early decelerations, late decelerations, and accelerations in simulated FHR images. Static images of simulated FHR tracings were created and each image was presented with and without the presence of a visual aid. Results showed that the visual aid helped observers increase correct detections overall. Among the novice observers in this study, the presence of the aid led to a higher rate of detections of early decelerations, late decelerations, and accelerations in FHR images $(M=0.62, \mathrm{SE}=0.03)$ compared with the condition in

Table 3 Signal type $\times$ variability interaction for proportion of correct detections, analyzed using a Bonferroni-Sidak test

\begin{tabular}{|l|l|l|l|}
\hline Signal type & Variability & Mean & Standard error \\
\hline Late Decel & Absent & 0.71 & 0.06 \\
\cline { 2 - 4 } & Minimal & 0.84 & 0.04 \\
\cline { 2 - 4 } & Moderate & 0.63 & 0.04 \\
\cline { 2 - 4 } & Marked & 0.36 & 0.05 \\
\hline Early Decel & Absent & 0.77 & 0.06 \\
\cline { 2 - 4 } & Minimal & 0.86 & 0.04 \\
\cline { 2 - 4 } & Moderate & 0.67 & 0.05 \\
\cline { 2 - 4 } & Marked & 0.39 & 0.05 \\
\hline Accel & Absent & 0.94 & 0.03 \\
\cline { 2 - 4 } & Minimal & 0.88 & 0.04 \\
\cline { 2 - 4 } & Moderate & 0.61 & 0.05 \\
\cline { 2 - 4 } & Marked & 0.32 & 0.04 \\
\hline
\end{tabular}

which no aid was overlaid on the images $(M=0.53$, SE $=0.03$ ). The aid was also useful in reducing false alarm rate specifically for images with marked variability.

It was expected that the presence of the visual aid would improve correct detection of maternal-fetal heart rate signals compared with trials in which no aid was present. This hypothesis was also supported. Proportions of correct detections were significantly higher when the aid was present than when the aid was absent. However, correct detection performance was still relatively poor. One reason for this low performance is that participants may have correctly identified the type of signal but incorrectly identified its magnitude. In the program developed for this study, participants selected a response choice that identified a type of signal (early deceleration, late deceleration, or acceleration) as well as its magnitude (small, medium, large), so there was no way for researchers to identify signals that were correctly identified independent of magnitude.

It was also expected that, based on previous research, ${ }^{7}$ detection performance would decrease as FHR variability increased. This hypothesis was supported. There was a significant effect of FHR variability on correct detections, such that means were highest for absent and minimal variability, lower for moderate variability, and lowest for marked variability.

Finally, it was expected that the visual aid would be most useful for FHR images with higher variabilities (moderate and marked). This prediction was based on the fact that higher FHR variability creates more "noise" that masks the visual signal. This hypothesis was partially supported. Although there was no significant interaction for correct detections between FHR variability and aid presence, there was indeed a significant interaction for false alarm rate between variability and aid presence. A test of simple effects revealed that the effect of aid was limited to marked variability. That is, false alarms were significantly lower (i.e., false alarm performance was better) in the marked variability condition when the aid was present, which presumably reduced participants' uncertainty as to the presence of a signal. This finding suggests that the aid was indeed more useful for signals embedded in marked variability, where detection was most difficult. 


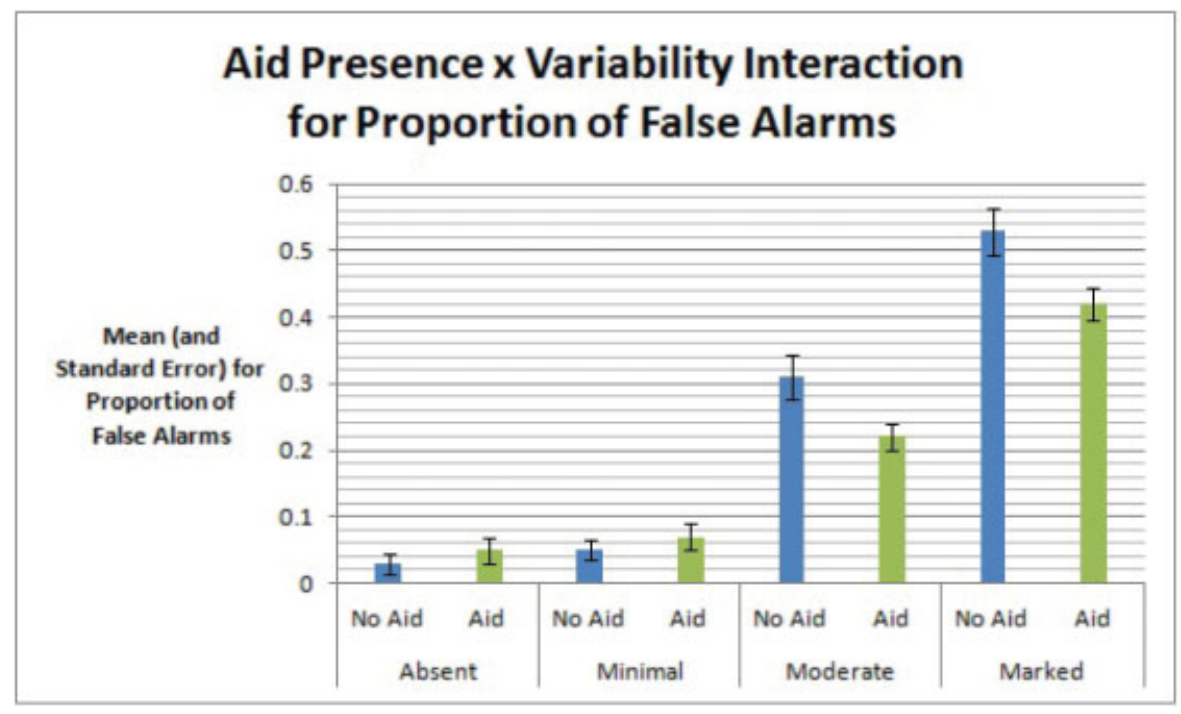

Fig. 4 Aid presence $\times$ variability interaction for false alarm rate.

Research in the psychology literature indicates that visual cues can help direct an observer's attention in visual tasks, thereby increasing accuracy and decreasing response time. ${ }^{8,9}$ A visual aid for FHR monitoring has been considered previously. Hall and colleagues ${ }^{10}$ created a prototype electronic ruler to assist with assessment of FHR variability. ${ }^{2}$ This electronic ruler contained a horizontal line placed at FHR baseline and a series of color-coded horizontal bands highlighting different levels of variability. Six obstetrics physicians assessed FHR variability on 10-minute tracings using either paper strips or the electronic ruler. The electronic ruler produced better assessment of variability than the paper strips, relative to the computer-calculated "gold standard." The findings from the present research extend these findings: whereas Hall et al used a visual aid to assist with observer identification of FHR variability, the current research applied a visual aid to facilitate the detection of important deviations from the FHR baseline.

The present study has some limitations. First, the sample was drawn from a naive undergraduate student population. The results will need to be replicated with trained professionals to determine whether the same pattern of results would be found with a clinical population. Second, participants viewed static images for a fixed interval rather than inspecting a dynamic tracing for a duration of their own choosing. Results may differ when participants have control over the image inspection time. A third limitation concerns the simulated FHR tracings. The images used in this study were generated specifically to impose greater experimental control over the signal characteristics. The results obtained here might differ with tracings derived from genuine patients. In fact, the present findings might paint a more optimistic picture of performance because all of the simulated tracings had a stable baseline and were free of variable decelerations and signal dropouts often found in genuine FHR tracings. Last, the signals in this study were defined as decelerations and accelerations without concern for the clinical importance of the patterns depicted in the tracings. The goal of the study was to examine the benefit of using visual aids on the perceptibility of significant deviations in FHR from the baseline. We believe this is a necessary first step before one can address the potential of visual aids for clinically significant patterns within FHR tracings.

In sum, the present findings suggest that the use of a visual aid could be a step toward improving clinicians' identification of important signals in FHR tracings. The implications of this line of research are that the presence of a visual aid may improve the ability of clinicians to properly interpret FHR patterns under conditions in which signals are difficult to identify, such as marked FHR variability.

\section{References}

1 Miller DA, Miller LA. Electronic fetal heart rate monitoring: applying principles of patient safety. Am J Obstet Gynecol 2012; 206(4):278-283

2 Macones GA, Hankins GD, Spong CY, Hauth J, Moore T. The 2008 National Institute of Child Health and Human Development workshop report on electronic fetal monitoring: update on definitions, interpretation, and research guidelines. Obstet Gynecol 2008;112(3):661-666

3 Belfore LA II, Scerbo MW, Anderson BL. A fetal heart rate monitor simulator. In: Proceedings from ModSim World September 2004; Virginia Beach, VA

4 Anderson BL, Scerbo MW, Belfore LA II, Abuhamad A. When is a deceleration perceived as a late deceleration? Simul Healthc 2010; 4:311

5 Anderson BL, Scerbo MW, Belfore LA II, Abuhamad AZ, Davis SS. Can clinicians tell when a decel is a late decel? In: 11th International Meeting on Simulation in Healthcare. New Orleans, LA: Society for Simulation in Healthcare; 2011

6 Kennedy RA, Anderson-Montoya BL, Scerbo MW, et al. The influence of visual aids on detecting early and late decelerations in maternal-fetal heart rate patterns. In: Proceedings of the Human Factors and Ergonomics Society 56th Annual Meeting. Boston, MA: Human Factors and Ergonomics Society; 2012:1317-1321 
e120 Crosshair Visual Aid and Detection of FHR Signals Kennedy et al.

7 Anderson BL, Scerbo MW, Belfore LA II, Abuhamad A. Detecting critical patterns in maternal-fetal heart rate signals. In: Proceedings of the Human Factors and Ergonomics Society 52nd Annual Meeting. Santa Monica, CA: Human Factors and Ergonomics Society; 2008:1478-1482

8 Chaney FB, Teel KS. Improving inspector performance through training and visual aids. J Appl Psychol 1967;51(4):311-315
9 Posner ML, Nissen MJ, Ogden WC. Attended and unattended processing modes: the role of set for spatial location. In: Pick JHI, Saltzman E, eds. Modes of perceiving and processing information. Hillsdale, NJ: Erlbaum; 1978:137-157

10 Hall LM, Hannon DJ, Leisk GG, Wolfberg AJ, House MD. Measurement of fetal heart rate variability on an electronic monitor using a prototype electronic ruler. Am J Perinatol 2012;29(6):409-413 